\title{
Breaking of equipartition in one-dimensional heat-conducting systems
}

\author{
Akiko Kato ${ }^{1}$ and David Jou ${ }^{2,3}$ \\ ${ }^{1}$ Institut für Theoretische Physik, Technische Universität Berlin, PN7-1, Hardenbergstrasse 36, D-10623 Berlin, Germany \\ ${ }^{2}$ Departament de Física, Universitat Autònoma de Barcelona, 08193 Bellaterra, Catalonia, Spain \\ ${ }^{3}$ Institut d'Estudis Catalans, Carme 47, 08001 Barcelona, Catalonia, Spain
}

(Received 16 April 2001; published 17 October 2001)

\begin{abstract}
Using information-theoretical methods, we studied how energy equipartition is broken in one-dimensional systems under a heat flow composed of alternating particles of two different masses. The average energy stored in particles of different masses is seen to be different in both ideal gases and harmonic lattices.
\end{abstract}

DOI: 10.1103/PhysRevE.64.052201

PACS number(s): 44.10.+i, 05.60.-k, 05.70.Ln

Much attention is currently being paid to heat transport in one-dimensional systems by using computer simulations of lattices or gases to examine the behavior of the heat flux as a function of the number of particles and, in particular, its behavior in the thermodynamic limit, i.e., when the number of particles tends to infinity (see, for instance, [1-4]). These analyses open many possibilities for the exploration and understanding of systems in nonequilibrium states and, in particular, of the thermodynamics of steady states and the microscopic foundations of Fourier's law. Two different situations are usually studied: all the particles are considered to have the same mass, or the mass of the different particles is considered as random. Very recently, two papers have been devoted to unidimensional gases composed of alternating particles with two different values of mass $[5,6]$. These two different values make the situation nonintegrable, in contrast with the case of one single value for the mass, and allow one to obtain significant results where the thermal conductivity in the thermodynamic limit is slowly divergent [5] or finite [6].

Here, we apply information theory [7-10] to two kinds of one-dimensional heat-conducting system, ideal gases and harmonic chains, with the aim of studying the possible breaking of equipartition of energy under the presence of a heat flux. With this objective in mind, we consider two kinds of particle with different masses and examine their average energy in nonequilibrium steady states, i.e., by imposing on the system constraints on the average total energy and total heat flux. Note that it is not assumed that the system does or does not satisfy Fourier's law, which is the central debated topic in one-dimensional systems; indeed, in contrast with the usual procedure, which imposes the values of the temperature at the boundaries and computes the heat flux for different numbers of particles, we focus our attention on a system with a given heat flux and compute the average values of the energy of the different kinds of particles. In our opinion, the wealth of recent results in the analysis of heat transport in one-dimensional systems could be used not only to study the conditions of validity of Fourier's law, and its convenient generalizations for microscopic systems, but also for the analysis of other topics of a more statistical nature, such as the breaking of local equilibrium or of equipartition.

We assume a maximum-entropy formalism with restrictions on the total energy and on the heat flux of the system. Since we consider a one-dimensional system in steady state, the heat flux must be the same everywhere and therefore the average contribution of each kind of particle to the heat flux must be the same. We thus consider a nonequilibrium distribution function of the canonical form

$$
f=Z^{-1} \exp \left[-\beta\left(H_{1}+H_{2}\right)-\gamma_{1} Q_{1}-\gamma_{2} Q_{2}\right],
$$

where $Z$ is the partition function ensuring normalization, and $\beta, \gamma_{1}$, and $\gamma_{2}$ are Lagrange multipliers imposing constraints on the average energy of the total system and on the heat flux through each subsystem, namely,

$$
\left\langle H_{1}+H_{2}\right\rangle=U, \quad\left\langle Q_{1}\right\rangle=\left\langle Q_{2}\right\rangle=Q=V q,
$$

where $V$ is the volume of the system and $q$ the heat transported per unit area and time (in one-dimensional systems, one has the length $L$ instead of $V$ and the heat flux has dimensions of energy divided by unit time).

We assume that both kinds of particle are intercalated in an alternating way throughout the system, in such a way that it is globally homogeneous. Accordingly, we impose the average value of the total energy only on each pair of particles, but not on each particle. Indeed, a thermometer put in contact with the system at any point would interact with one particle of each kind, and would indicate only one temperature. In contrast, if we supposed that the system were composed of two homogenous subsystems, each of them formed by particles of the same kind, it would seem more logical to impose constraints on the energy of each subsystem, because a thermometer at one point would interact only with particles of one kind. In both situations, however, the value of the heat flux must be the same for both kinds of particle, due to the energy balance condition in the steady state. Our question refers to the proportion of energy stored in each kind of particle in the presence of a heat flux.

Let us mention that one could interpret this study in two different ways: as an analysis of the system as a whole as a discrete thermodynamic system, with $\beta$ being a global parameter such as the so-called contact temperature [11], or as a local analysis concentrated on a given pair of particles. In this case, the coefficient $\beta$ would have a local character which could make it closer to the temperature indicated by a thermometer at a given point of the system.

(a) Ideal gas in one dimension. We first consider an ideal nonrelativistic gas at rest under fixed values of energy and heat flux in one dimension. The distribution function when 
all the particles have the same mass $m$ is given, according to the maximum-entropy formalism, by

$$
f=Z^{-1} \exp \left[-\beta \frac{1}{2} m C^{2}-\gamma\left(\frac{1}{2} m C^{2}-\frac{3}{2} \beta^{-1}\right) C\right],
$$

with $m$ the mass of the particles. Here we have taken into account that the microscopic operators for the energy and the heat flux are $(1 / 2) m C^{2}$ and $(1 / 2) m C^{2} \mathcal{C}$, with $\mathcal{C}$ the specific velocity of the particle with respect to the barycentric velocity; finally, the term $\frac{3}{2} \beta^{-1} C$ guarantees that the average velocity of the system is zero. Usually, one expands Eq. (3) up to second order in $\gamma$ as

$$
\begin{aligned}
f= & Z^{-1} \exp \left(-\beta \frac{1}{2} m C^{2}\right)\left[1-\gamma\left(\frac{1}{2} m C^{2}-\frac{3}{2} \beta^{-1}\right) C\right. \\
& \left.+\frac{1}{2}(\gamma C)^{2}\left(\frac{1}{2} m C^{2}-\frac{3}{2} \beta^{-1}\right)^{2}\right] .
\end{aligned}
$$

The partition function $Z$ ensures the normalization of the distribution function and is found to be

$$
\begin{aligned}
Z & =\int_{-\infty}^{+\infty} \exp \left(-\beta \frac{1}{2} m C^{2}\right)\left[1+\frac{1}{2}(\gamma C)^{2}\left(\frac{1}{2} m C^{2}-\frac{3}{2} \beta^{-1}\right)^{2}\right] d C \\
& =\sqrt{2 \pi / m \beta}\left[1+\frac{3}{4 m \beta^{3}} \gamma^{2}\right]
\end{aligned}
$$

whereas the integral of the term of first order in $\gamma$ vanishes because it is odd in $\mathcal{C}$.

The average value of the energy is given by the integral of $(1 / 2) m C^{2}$ and yields

$$
\frac{U}{N}=\frac{1}{2 \beta} \frac{1+\left(21 / 4 m \beta^{3}\right) \gamma^{2}}{1+\left(3 / 4 m \beta^{3}\right) \gamma^{2}} \approx \frac{1}{2 \beta}\left[1+\frac{9}{2 m \beta^{3}} \gamma^{2}\right],
$$

where $N$ is the number of particles in the system. The average value of the heat flux is given by the integral of $(1 / 2) m C^{2} \mathcal{C}$ over the distribution function and is given by

$$
q=-\frac{\left(6 / 4 m \beta^{3}\right)(N / L) \gamma}{1+\left(3 / 4 m \beta^{3}\right) \gamma^{2}} \approx-\frac{6}{4 m \beta^{3}} \frac{N}{L} \gamma,
$$

where $L$ is length of the system. Combination of Eqs. (6) and (7) allows us to write

$$
\frac{U}{N}=\frac{1}{2 \beta}\left[1+2 m \beta^{3} \frac{L^{2}}{N^{2}} q^{2}\right] .
$$

Up to now, we have referred to particles of the same kind. Here, we want to deal with a one-dimensional gas composed of two kinds of particle, of masses $m_{1}$ and $m_{2}$, respectively. Instead of imposing a priori a value for the internal energy associated with each kind of particle, we only fix the total energy. Then, using expression (6) for both kinds of particles, we get

$$
\frac{U}{N}=\frac{1}{2 \beta}\left[1+\frac{9}{2 m_{1} \beta^{3}} \frac{N_{1}}{N} \gamma_{1} \gamma_{1}+\frac{9}{2 m_{2} \beta^{3}} \frac{N_{2}}{N} \gamma_{2} \gamma_{2}\right]
$$

with $N_{1}$ and $N_{2}$ the respective numbers of particles 1 and 2 . Note that there is only one value of $\beta$ but two values of $\gamma$ because we are imposing only the total value of the energy. Concerning the Lagrange multipliers conjugated to the heat flux, we take into account that the heat flux must be equal for both kinds of particle because they are in series in the onedimensional system. Thus, in view of Eq. (7) one has

$$
q=-\frac{3}{2 m_{1} \beta^{3}} \frac{N_{1}}{L_{1}} \gamma_{1}=-\frac{3}{2 m_{2} \beta^{3}} \frac{N_{2}}{L_{2}} \gamma_{2},
$$

where $L_{1}$ and $L_{2}$ are, respectively, the lengths of the system occupied by particles 1 and 2 . Furthermore, it must be taken into account that the equality of pressure imposes that $\left(N_{1} / L_{1}\right) \beta^{-1}=\left(N_{2} / L_{2}\right) \beta^{-1}$.

Equations (9) and (10) determine the values of $\beta, \gamma_{1}$, and $\gamma_{2}$ in terms of $U$ and $q$. Now, we ask how the total internal energy (9) is distributed in both kinds of particle. By combining Eqs. (9) and (10) we obtain

$$
\frac{u_{1}}{u_{2}}=\frac{U_{1} / N_{1}}{U_{2} / N_{2}}=\frac{1+2 m_{1} \beta^{3}\left(L_{1}^{2} / N_{1}^{2}\right) q^{2}}{1+2 m_{2} \beta^{3}\left(L_{2}^{2} / N_{2}^{2}\right) q^{2}},
$$

where $u$ stands for the energy per particle. This expression yields the conclusion that in the presence of a heat flux the heavier particles have more energy, on the average, than the lighter ones, if the number of particles per unit length is the same for both species. Of course, when the heat flux vanishes one recovers the classical equipartition.

(b) Harmonic lattice. The second system we will consider is a harmonic chain. In harmonic chains, the phonon mean free path is infinite and, consequently, the energy flux is not proportional to the temperature gradient but to the temperature difference between the reservoirs located at its ends. To avoid complications associated with the boundary conditions, Miller and Larson [12,13] eliminate the boundaries by considering a ring where, because of its infinite heat conductivity, a heat flux lasts indefinitely. Such a chain ring is characterized by constraints on the average internal energy $U$ and the average heat flux $Q$.

The system we consider consists of a harmonic chain of $N$ particles, $N / 2$ of mass $m_{1}$ and $N / 2$ of mass $m_{2}$, intercalated with each other in order to have, as in the previous situation, a globally homogeneous system. Each particle is connected to its nearest neighbors by Hookean springs with stiffness $\kappa$. Let $q_{i}$ be the displacement from equilibrium for each particle $i$ and $p_{i}$ its conjugate momentum. The Hamiltonian $\mathcal{H}\left(q_{1}, p_{1}, \ldots, q_{N}, p_{N}\right)$ is given by

$$
\mathcal{H}=\frac{1}{2} \sum_{i} \frac{p_{i}^{2}}{m_{i}}+\frac{1}{2} \sum_{i} \kappa\left(q_{i+1}-q_{i}\right)^{2}
$$

and the microscopic operator $\mathcal{J}\left(q_{1}, p_{1}, \ldots, q_{N}, p_{N}\right)$ for the heat flux is [10]

$$
\mathcal{J}=-\frac{1}{2} \sum_{i} \frac{\kappa}{m_{i}}\left(q_{i+1} p_{i}-q_{i} p_{i+1}\right) .
$$

First of all, we summarize the results obtained in [13] for a chain composed of particles having the same mass. One assumes as in Eq. (1) a distribution function of the form 


$$
f=Z^{-1} \exp (-\beta \mathcal{H}-\gamma \mathcal{J})
$$

By transforming $\mathcal{H}$ and $\mathcal{J}$ in terms of normal coordinates it follows that [13]

$$
Z=\left\{\frac{1}{2} \beta\left[1+\left(1-y^{2}\right)^{1 / 2}\right]\right\}^{-N},
$$

where $y$ is the dimensionless ratio $y=(\sqrt{\kappa / m}) \gamma / \beta$. The Lagrange multipliers $\beta$ and $\gamma$ may be found in terms of $U$ and $Q$ through the constraints

$$
U=\langle\mathcal{H}\rangle=\frac{\partial \ln Z}{\partial \beta}, \quad Q=\langle\mathcal{J}\rangle=-\frac{\partial \ln Z}{\partial \gamma},
$$

which yield

$$
\begin{aligned}
& \frac{U}{N}=\frac{1}{\beta} \frac{1+\left(1-y^{2}\right)^{1 / 2}}{1-y^{2}+\left(1-y^{2}\right)^{1 / 2}} \approx \frac{1}{\beta}\left[1+\frac{1}{2} y^{2}\right], \\
& q=-\frac{1}{\beta} \frac{y}{1-y^{2}+\left(1-y^{2}\right)^{1 / 2}} \approx-\frac{y}{2 \beta} \sqrt{\kappa / m} .
\end{aligned}
$$

By combining Eqs. (17) and (18), we may write for the energy

$$
\frac{U}{N}=\frac{1}{\beta}\left[1+2 \beta^{2} \frac{m}{\kappa} q^{2}\right] .
$$

As in the previous example of the ideal gas, we consider now particles with two different masses, assuming that only the total energy is fixed, and that the heat flux crossing each position must be the same. We finally obtain, by following the same arguments as in the previous case,

$$
\frac{u_{1}}{u_{2}}=\frac{U_{1} / N_{1}}{U_{2} / N_{2}}=\frac{1+2 \beta^{2} m_{1} \kappa^{-1} q^{2}}{1+2 \beta^{2} m_{2} \kappa^{-1} q^{2}} .
$$

Again, it is found that the heavier particles have higher energy in the presence of the heat flux, and the classical equipartition result is recovered when the heat flux vanishes.

The distribution function used here could be applied to the study of the second moments of the fluctuations of the energy and the heat flux, which will be affected by the presence of the nonvanishing heat flux. This may have interest, for instance, in the application of the fluctuation-dissipation theorem, but we have preferred to focus our attention on an explicit illustration of the breaking of equipartition.

Breaking of equipartition in nonequilibrium situations is to be expected, although it is usually not studied explicitly. Here, we have tried to go beyond more or less intuitive expectations (which are not able to say, for instance, whether the lighter or the heavier particles should have more energy) and have undertaken a quantitative analysis. For instance, in previous work $[12,14]$ we showed that informationtheoretical methods yield the conclusion that in an ideal gas under a heat flux the average kinetic energy of the particle is different in the directions normal to the heat flux than in the direction along it, in such a way that if the heat flux is directed along the $y$ direction, one has

$$
\left(\frac{1}{2} m \nu_{x}^{2}\right)=\left\langle\frac{1}{2} m \nu_{z}^{2}\right\rangle<\frac{1}{2} k_{B} T, \quad\left\langle\frac{1}{2} m \nu_{y}^{2}\right\rangle>\frac{1}{2} k_{B} T .
$$

These trends have been qualitatively observed in independent analyses based on nonequilibrium molecular dynamics [15].

The present situation provides another illustration of this breaking, which is of special interest because of the large number of recent simulations of one-dimensional heatconducting systems. We are aware of only two recent papers studying unidimensional gases composed of alternating particles with two different masses $[5,6]$. Both papers deal mostly with their dynamical aspects (the system is nonintegrable, in contrast with systems with only one value of the mass) and obtain a slowly divergent [5] or even a finite value [6] for the thermal conductivity. One of these papers [5] does not specify how energy is distributed in the two kinds of particle. The other indicates that the lighter particles tend to absorb more energy than the heavier ones, in contrast with our results. We do not know, at present, the origin of this discrepancy, and one of the aims of our report is precisely to encourage such research. Indeed, there are several possibilities for this disagreement: the fact that in the usual papers the value of the temperature is imposed on the boundaries and the heat flux is calculated a posteriori, whereas here we impose a given value for the heat flux; or it could be the uncertainty as to which ensemble should be used in the analysis of situations in the presence of a heat flux, or the breaking of ergodicity, or the limitations of the maximum-entropy method, etc. At this moment, having only one numerical result for gases and not a single result for lattices, it is premature to speculate about any conclusion on this topic, which we consider as open (note, for instance, that although Refs. [5] and [6] deal with particles with two masses, in Ref. [6] thermal conductivity is finite in the thermodynamic limit, but it diverges slowly in Ref. [5]).

Two other points to be noted are the following. (1) The present analysis does not discuss whether Fourier's law is satisfied or not, i.e., whether the heat flux is proportional to $\Delta T / L$ or to $\Delta T / L^{\alpha}$, with $\alpha$ a coefficient different from $1, \Delta T$ being the difference of the temperatures of the heat reservoirs at the ends of the system. Our analysis uses only the microscopic expression for the heat flux and the condition of steady state; thus, it could be useful to analyze the results of computer simulation techniques, commonly used in this field with the aim of determining the behavior of the system as the number of particles is changed, when two different kinds of particle are considered. For instance, for harmonic chains, the energy current is a conserved quantity, in such a way that some authors [13], instead of imposing boundary conditions for the temperature, assumed a ring chain where the energy flux is conserved, as well as the energy, thus having these two quantities as natural constraints on the system in an information-theoretical analysis. (2) It is noted that the Lagrange multiplier conjugated to the Hamiltonian is no longer the inverse of the local equilibrium temperature $[12,14]$, but a more general function that depends not only on the energy but also on the heat flux, as can be seen, for instance, from Eqs. (8) and (11) for the two kinds of systems 
we have considered. This could be of interest in the analysis of heat transport when the value of the heat flux is high.

We want to mention finally that other kinds of breaking of equipartition in one-dimensional systems have been pointed out in particles of the same mass but with inelastic collisions [16]. It is observed that a few particles concentrate most of the internal energy of the system, whereas the other particles form a kind of aggregate and stay essentially at rest. We cannot apply the present analysis to this interesting situation because in the presence of inelastic collisions the heat flux is no longer constant throughout the system, since a part of the energy is dissipated in each collision. Thus, one should consider separately the energy transported along the system by collisions between particles and the energy dissipated in the collisions.

A.K. acknowledges the support of the Helene und Erwin Stephan-Stiftung of the Technische Universität Berlin. D.J. acknowledges the financial support of the Dirección General de Investigación Científica y Técnica of the Spanish Ministry of Science and Technology under Grant No. BFM20000351-C03-01 and of the Direcció General de Recerca of the Generalitat of Catalonia under Grant No. 1999SGR00095.
[1] S. Lepri, R. Livi, and A. Politi, Phys. Rev. Lett. 78, 1896 (1997); Europhys. Lett. 43, 271 (1998); Physica D 119, 140 (1998).

[2] C. Giardina, R. Livi, A. Politi, and M. Vassalli, Phys. Rev. Lett. 84, 2144 (2000); T. Prosen and D. Campbell, ibid. 84, 2857 (2000); O. V. Gendelman and A. V. Savin, ibid. 84, 2381 (2000).

[3] T. Hatano, Phys. Rev. E 59, R1 (1999); B. Hu, B. Li, and H. Zhao, ibid. 61, 3828 (2000).

[4] H. Aoki and D. Kusnezov, Phys. Lett. B 477, 348 (2000); H. Kaburaki and M. Machida, Phys. Lett. A 181, 85 (1993).

[5] A. Dhar, Phys. Rev. Lett. 86, 3554 (2001).

[6] P. L. Garrido, P. I. Hurtado, and B. Nadrowski, Phys. Rev. Lett. 86, 5486 (2001).

[7] R. Luzzi, A. R. Vasconcellos, and J. Galvao-Ramos, Statistical Foundations of Irreversible Thermodynamics (TeubnerVerlag, Berlin, 2000).
[8] D. N. Zubarev, V. Morozov, and G. Röpke, Statistical Mechanics of Nonequilibrium Processes (Akademie-Verlag, Berlin, 1997).

[9] W. T. Grandy, Jr., Phys. Rep. 62, 175 (1980); Foundations of Statistical Mechanics, Vols. 1 and 2 (Reidel, Dordrecht, 1988).

[10] The Maximum Entropy Formalism, edited by R. D. Levine and M. Tribus (MIT Press, Cambridge, MA, 1979).

[11] W. Muschik, Arch. Ration. Mech. Anal. 66, 379 (1977).

[12] D. Jou, J. Casas-Vázquez, and G. Lebon, Extended Irreversible Thermodynamics, 2nd ed. (Springer, Berlin, 1997).

[13] B. N. Miller and P. M. Larson, Phys. Rev. A 20, 1717 (1979).

[14] J. Camacho and D. Jou, Phys. Rev. E 52, 3490 (1995); J. Casas-Vázquez and D. Jou, ibid. 49, 1040 (1994).

[15] G. P. Morriss and L. Rondoni, Phys. Rev. E 59, R5 (1999); A. Baranyai, ibid. 61, R3306 (2000); 62, 5989 (2000).

[16] Y. Du, H. Li, and L. P. Kadanoff, Phys. Rev. Lett. 74, 1268 (1995). 